Musa S. Danazumi*, PT, MScPT, Abdulsalam M. Yakasai, PT, PhD, Aminu A. Ibrahim, PT, PhD, Usman T. Shehu, PT, MPH and Shehu U. Ibrahim, PT, MScPT

\title{
Effect of integrated neuromuscular inhibition technique compared with positional release technique in the management of piriformis syndrome
}

https://doi.org/10.1515/jom-2020-0327

Received December 26, 2020; accepted March 29, 2021;

published online May 31, 2021

\section{Abstract}

Context: Studies have indicated that the muscle energy technique (MET) and the positional release technique (PRT) are effective in the management of piriformis syndrome (PS); however, evidence is scarce regarding the combination of these techniques in the form of an integrated neuromuscular inhibition technique (INIT) in the management of individuals with PS. Although a previous trial investigated the effect of INIT for PS, that study did not integrate Ruddy's reciprocal antagonist facilitation (RRAF) method into the INIT protocol, nor did the authors diagnose PS according to established criteria.

Objectives: To examine the effects of INIT with integrated RRAF compared with PRT in the management of patients diagnosed with PS.

\footnotetext{
*Corresponding author: Musa S. Danazumi, PT, MScPT, Department of Physiotherapy, Federal Medical Center, Nguru, 630101 Machina Road, Nguru, Yobe State, Nigeria, E-mail: musadanazumisani@gmail.com. https://orcid.org/0000-0002-3084-5552

Abdulsalam M. Yakasai, PT, PhD, Medical Rehabilitation Therapists (Reg.) Board of Nigeria, North-West Zonal Office, Kano, Nigeria; and Department of Physiotherapy, College of Health Sciences, University of KwaZulu-Natal, Durban, South Africa. https://orcid.org/00000001-8091-5443

Aminu A. Ibrahim, PT, PhD, Department of Physiotherapy, Faculty of Allied Health Sciences, College of Health Sciences, Bayero University, Kano, Nigeria; and Department of Physiotherapy, Muhammad Abdullahi Wase Teaching Hospital, Hospitals Management Board, Kano, Kano State, Nigeria. https://orcid.org/0000-0002-5711-1639 Usman T. Shehu, PT, MPH, Department of Public Health, School of Health Sciences, University of Salford Manchester, Manchester, UK Shehu U. Ibrahim, PT, MScPT, Department of Physiotherapy, Faculty of Allied Health Sciences, College of Health Sciences, Bayero University, Kano, Nigeria
}

Methods: This study was designed as a single blind randomized clinical trial in which participants diagnosed with PS were randomly allocated into INIT and PRT groups. Each group attended two treatment sessions per week for 8 weeks. Patients in the INIT group received a protocol in which the patient's tender point or trigger point was palpated in the belly of the piriformis approximately halfway between the inferior lateral angle of the sacrum and the greater trochanter, at which point the therapist applied an intermittent or sustained pressure and maintained the pressure for 20-60 seconds (depending on the participant's response to pain reduction). For INIT patients, that protocol was also followed by RRAF, a method in which a patient introduces a series of tiny/miniature contractions or efforts (20 times per 10 seconds) against a therapist's resistance. Patients in the PRT group were treated by palpating the same trigger point described in the INIT group, followed by application of light pressure at the location of the trigger point, which was maintained the pressure for 2 minutes or until the pain subsided (determined by asking the participant to report a pain score using a visual analog scale at 30 second intervals). For both groups, three repetitions of the INIT or PRT treatment were performed over 10 minutes at each clinical visit. Additionally, each group also received stretching exercises immediately after the INIT or PRT treatment session. Each participant was assessed at baseline, immediately posttreatment, and at 4 months posttreatment for pain, sciatica, functional mobility, quality of life, hip abduction, and internal rotation. A repeated measures analysis of variance (ANOVA) of within-between group interactions was used to analyze the treatment effect.

Results: Forty eight participants (age range, 25-47 years; mean age \pm standard deviation, $32.81 \pm 3.27$ years) were randomized into the INIT and PRT groups, with 24 participants in each group. No significant between-group differences ( $p>0.05$ ) were observed in the baseline demographic 
and clinical variables of the participants. A repeatedmeasures ANOVA indicated that there was a significant time effect for all outcomes, with a significant interaction between time and intervention ( $\mathrm{p}<0.001)$. The Bonferroni post hoc analyses of time and intervention effects indicated that the INIT group improved significantly compared with the PRT group in all outcomes $(\mathrm{p}<0.05)$ immediately posttreatment and at the 4 months follow up period.

Conclusions: INIT was more effective than PRT in the management of individuals with PS. It should be noted the significant improvement achieved in both the groups may have also been contributed to by the stretching exercises that were used as adjunct therapies by both groups.

Keywords: integrated neuromuscular inhibition technique; muscle energy technique; piriformis syndrome; positional release technique.

Piriformis syndrome (PS) is a neuromuscular condition characterized by a constellation of symptoms that includes hip and buttock pain [1]. The pain is often referred down the back of the leg, and sometimes into the medial foot [2]. Though similar in presentation to a true L5 or S1 radiculopathy, this peripheral neuritis is presumed to be the result of an abnormal piriformis muscle, compression, or irritation of the sciatic nerve as it travels under or through the muscle [1, 2]. Given its similar presentation to lumbar disc herniation with radiculopathy, stenosis with radiculopathy, and neurogenic pain, PS is often missed or difficult to diagnose [2]. In a 2013 study of 2,910 patients with low back/buttock pain with sciatica, 183 (6.25\%) had PS [3]. Delay in diagnosing PS may lead to pathologic conditions of the sciatic nerve, chronic somatic dysfunction, and compensatory changes resulting in pain, paresthesia, hyperesthesia, and muscle weakness [4].

Treatment options for PS focus on the subjective and objective findings of the assessment [5]. In most circumstances, an inflammatory response is suspected in the muscle or sciatic nerve; therefore, the initial treatment goals involve decreasing inflammation, associated pain, and spasm [5, 6]. Treatment options may include pharmacology $[7,8]$, osteopathic manipulation $[9,10]$, physical therapy $[11,12]$, injection therapy $[13,14]$, and surgical decompression [15-17]. Notably, no single treatment option has been proven most effective in the management of PS and a previous systematic review [8] indicated a lack of effective nonoperative management techniques to help ameliorate this most commonly misdiagnosed condition.

The positional release technique (PRT) has been investigated as an effective indirect osteopathic manipulative treatment in the management of individuals with PS
[9]. This technique involves the identification of active trigger points, followed by the application of ischemic compression to reproduce the nociceptive response. The limb is then positioned to reduce the tension in the affected muscle and subsequently the painful trigger points are deactivated [18]. One proposed mechanism for the benefit of ischemic compression was explained by Hou et al. [19], who suggested that pain and muscle spasm relief from direct digital pressure may result from the reactive hyperemia produced in the area or from the spinal reflex mechanism.

Alternately, Chaitow et al. [20] posited that an integrated neuromuscular inhibition technique (INIT), a combination of the muscle energy technique (MET) and PRT, may produce the most effective approach to treating trigger points. They hypothesized that the benefit of the technique lies in its multifaceted approach, which allows for the delivery of the techniques in a single coordinated manner. Although randomized clinical trials have examined the effects of MET [10, 11, 21, 22] and PRT [9] in the management of piriformis syndrome, the evidence is limited regarding the integration of these techniques in the form of INIT for PS. While an earlier study [23] investigated the effect of INIT in the management of PS, it had limitations. That study [23] reported very short term follow up data (immediately after one treatment session) and did not use valid diagnostic criteria for PS [5].

In this study, we examined the effectiveness of INIT compared with PRT in the management of patients with PS. We hypothesized that there would be no significant difference between INIT and PRT in the management of PS.

\section{Methods}

\section{Research design and ethics}

This study was designed as a single blind, randomized clinical trial; the research participants were blinded to randomization and group allocation, but the investigators were not blinded due to the nature of the study. However, outcomes assessors (research assistants) and data analysts were blinded. The guidelines set forth by the Declaration of Helsinki were followed. Ethical approval to conduct this study was obtained from the Health Research Ethics Committee of Federal Medical Centre (FMC), Nguru, Yobe State, Nigeria (FMC/N/CL.SERV/ 355/VOL IV/146). The trial was registered with the Pan African Clinical Trial Registry (PACTR201911583499287). Participants were assured of their rights to withdraw from the study at any point in time and their full anonymity was maintained, and they were asked to report any adverse events that occurred during the treatment period. The trial began on November 11, 2019 and ended on October 12, 2020. 


\section{Sample size and sampling technique}

The sample size was calculated using $G^{\star}$ Power version 3.1 software. The effect size used to calculate the sample size was obtained from a previous study [10] using pain as the outcome measure between two experimental groups. The calculation yielded a total sample size of 40 participants (20 per group) using the following inputs: tail (1), probability level $(\alpha-0.05)$, power $(p-0.8)$, effect size $(d-0.82)$, and allocation ratio (N2/N1-1) for t-test of difference between two independent means (two groups). According to a previous study [24], a 20\% attrition rate in a clinical trial should be accounted for to ensure equal participant allocation to groups. For this reason, the sample size was adjusted to 48 participants (24 per group) to account for attrition. The sampling method used to obtain the required number of participants in this study was consecutive. The consecutive sampling method was chosen because the participants were not all present at once.

\section{Eligibility criteria}

Male and female participants aged 19 years and older with leg or buttock pain diagnosed by one of two orthopedic physiotherapists (M.S.D., M.A.F.) as having chronic (3 months or longer) PS without any spinal pathology or involvement were eligible for enrollment in the study. The diagnostic criteria included: (1) unilateral buttock pain and radiculopathy due to spasms of the piriformis muscle or sciatic nerve compression; (2) buttock pain aggravated when sitting; (3) external tenderness near the greater sciatic notch and pain with any maneuver (e.g., pain with passive internal rotation of the hip [Freiberg sign]; provocation of sciatic symptoms by lifting and holding the affected leg 4 inches off the table when the participant lies on the unaffected leg (Beatty test); and reproduction of sciatic symptoms using a flexion, adduction, and internal rotation (FAIR) test performed with the patient in a lateral recumbent position with the affected side up, the hip flexed to an angle of $60^{\circ}$, the knee flexed to an angle of $60^{\circ}-90^{\circ}$ while stabilizing the hip, and the examiner internally rotating and adducting the hip by applying downward pressure to the knee) that increased piriformis muscle tension; and (4) limited straight leg raising ability [5].

Participants with the following were excluded: (1) psychiatric conditions that would have made it difficult to provide consent, as assessed by one author (M.S.D.) using Mini-Mental State Examinations; (2) any pathology or recent injury around the hip, sacroiliac joint, or lumbar spine; (3) limb length discrepancy; (4) recent buttock trauma and bladder/bowel dysfunction; or (5) deep gluteal syndrome (entrapment of sciatic nerve in the deep gluteal space) or extrapelvic compression of the sciatic nerve or sacral plexus including sciatic neuritis due to gamelli-obturator internus syndrome, compression of the pudendal nerves or increased mechanical stress on the innominate bones, compression of the fibular branch of the sciatic nerve, ischiogluteal/ischiofemoral bursitis or impingement, upper hamstrings tendinitis, or referred pain from gastrointestinal or pelvic causes (including colon cancer, endometriosis, and interstitial cystitis) [25-27].

\section{Data collection procedure}

Participant recruitment: Participants were recruited from the general and surgical outpatient departments of FMC Nguru, Nigeria to the physiotherapy department of the same hospital, using posters and verbal announcements made by one of the authors (M.S.D).
Participants attending the physiotherapy outpatient clinic at the same hospital were also recruited. Participants were screened for eligibility by orthopedic physiotherapists and those who met the eligibility criteria and were willing to participate were enrolled in the study. All participants signed a written informed consent after which their demographic and clinical parameters were obtained.

Outcomes assessment: Outcomes were assessed at baseline and immediately posttreatment. Treatment ended after 8 weeks of intervention and participants were given a regimen of stretching exercises to be performed at home. Outcomes were further assessed at the follow up visit 4 months posttreatment.

(1) Pain: Visual analog scale (VAS) was used to assess buttock/leg pain intensity. The assessment was based on a horizontal $10 \mathrm{~cm}$ scale varying from 1 (least pain in the leg) to 10 (the worst pain ever). VAS has been shown to have a high interrater reliability coefficient ( $\mathrm{r}=0.88)$ [28].

(2) Sciatica: The Sciatica Bothersomeness Index (SBI) was used to assess the level of sciatica among the participants. The scale's scores range from 0 to 24 , with higher scores reflecting severe sciatica bothersomeness. This scale has a high level of internal consistency reliability (Cronbach's alpha of 0.70) and test-retest reliability (intraclass correlation coefficient [ICC] of 0.90) [29]. A change of 6.5 points is considered clinically significant [29].

(3) Functional Mobility: Timed Up and Go (TUG) test was used to objectively measure functional mobility. This test was performed with a high back chair with armrests. Participants began seated and leaning back in the chair, then rose from the chair by using their arms for support following the command "Go." Participants walked $3 \mathrm{~m}$ in a straight line at their normal walking speed and then returned to the chair and sat down again. A stopwatch was used to measure the time taken to complete the task. TUG has been shown to have a high interrater reliability coefficient $(\mathrm{r}=0.99)$ and intraclass correlation coefficient ( $\mathrm{r}=0.97)$ [30].

(4) Quality of Life: This was assessed using the Short Form Health Survey (SF-36) questionnaire. The questionnaire consists of 36 items addressing physical and social functioning across eight domains: (1) physical functioning; (2) physical restrictions; (3) emotional restrictions; (4) social functioning; (5) somatic pain; (6) general mental health; (7) vitality; and (8) general health perception. The total score ranges from 0 to 100 , with higher scores indicating better quality of life and lower scores indicating the worse quality of life [31]. The reliability estimates for the SF-36 have been reported to have exceeded the recommended minimum value of 0.70 for internal consistency reliability, with figures typically over 0.80 for test-retest reliability [32].

(5) Hip Abduction: A simple long arm goniometer (Orthopedic Equipment Co.) with a $360^{\circ}$ scale marked in $1^{\circ}$ increments was used. The measurements were done with participants in the supine position. Hip abduction was measured from the neutral zero position in which the longitudinal axis of the thigh was perpendicular to the transverse line across the anterior superior iliac spine of the pelvis, which was used for alignment of the stationary arm of the goniometer. The unilateral anterior superior iliac spine was used to center the fulcrum of the goniometer and the moveable arm of the goniometer was aligned over the midline of the femur pointing at the center of the patella. Participants placed the contralateral leg hanging down from the edge of the couch to ensure that the pelvis did not move during the abduction 
(Figure 1). The concurrent validity and the test-retest reliability were good with ICCs of 0.94 and 0.92, respectively [33].

(6) Hip Internal Rotation: For hip internal rotation, participants (lying supine) had the hip and the knee flexed to $90^{\circ}$. The stationary arm of the goniometer was aligned parallel to the transverse line across the anterior superior iliac spines of the pelvis with the fulcrum of the goniometer centered over the patellar apex. Internal rotation was measured as the deviation from the starting position, in which the longitudinal axis of the leg was perpendicular to the transverse line across the anterior superior iliac spine (Figure 2). The concurrent validity and the test-retest reliability were good with ICCs of 0.88 and 0.95 , respectively [33].

\section{Participant randomization}

Eligible participants who provided informed consent were randomized into either the INIT or PRT group in a 1:1 ratio. A randomization

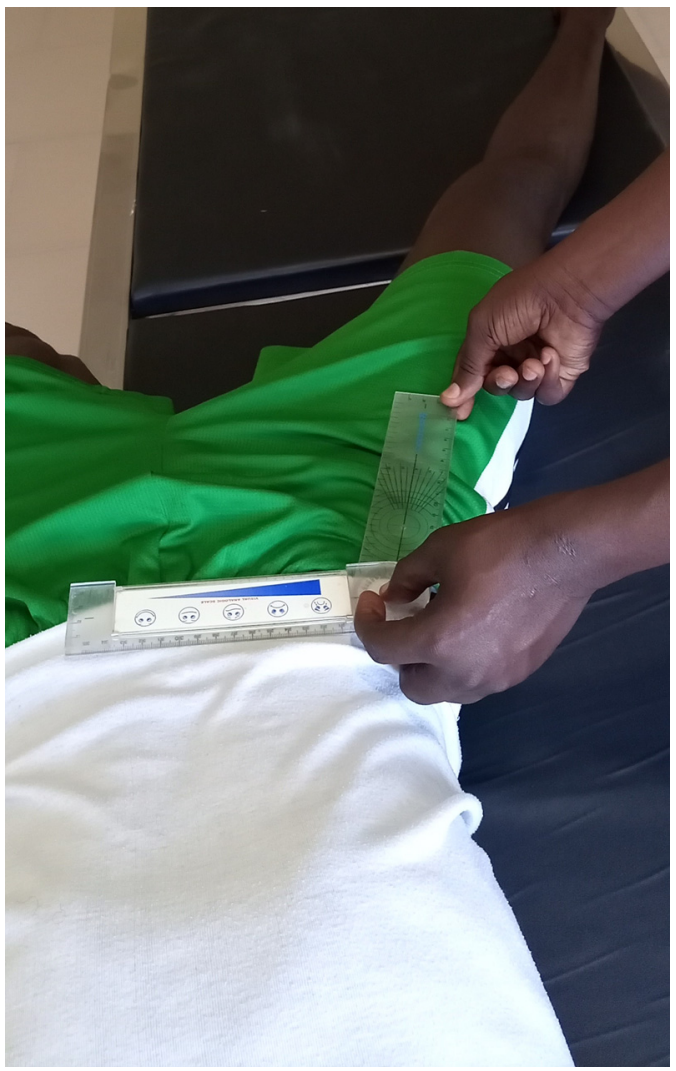

Figure 1: Hip abduction measurement is shown with the hip in abducted position. Initially, hip abduction was measured from the neutral zero position in which the longitudinal axis of the thigh was perpendicular to the transverse line across the anterior superior iliac spine of the pelvis; this was used for alignment of the stationary arm of the goniometer. The unilateral anterior superior iliac spine was used to center the fulcrum of the goniometer and the moveable arm of the goniometer was aligned over the midline of the femur pointing at the center of the patella. Participants placed the contralateral leg hanging down from the edge of the couch to ensure that the pelvis did not move during the abduction.

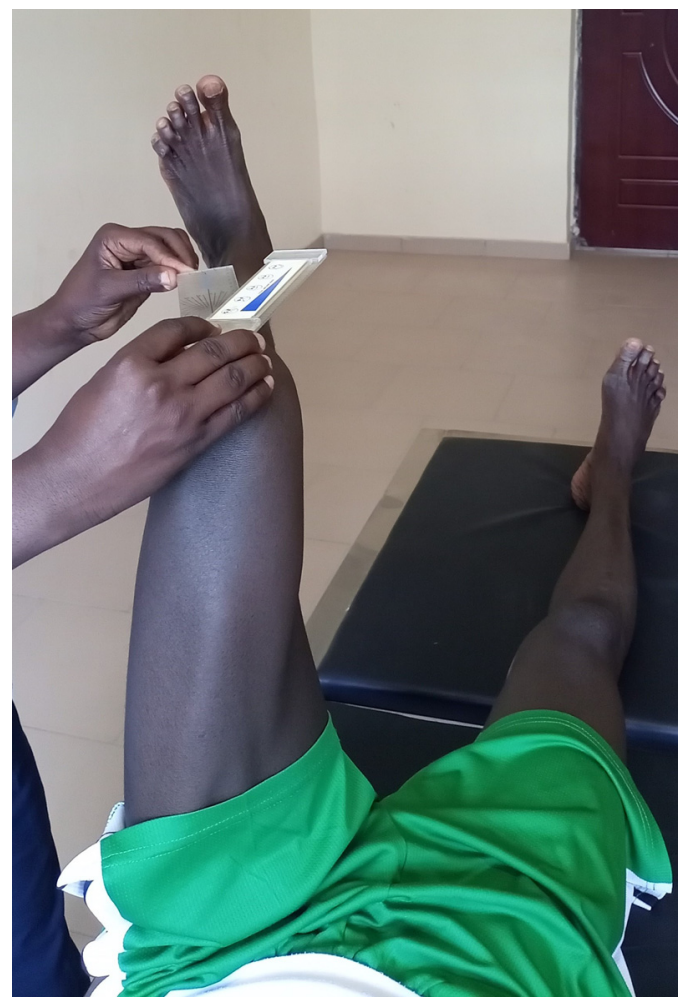

Figure 2: Hip internal rotation measurement is shown with the hip in internally rotated position. For this measurement, participants (lying supine) had the hip and the knee flexed to $90^{\circ}$. The stationary arm of the goniometer was aligned parallel to the transverse line across the anterior superior iliac spines of the pelvis with the fulcrum of the goniometer centered over the patella apex. Internal rotation was measured as the deviation from the starting position, in which the longitudinal axis of the leg was perpendicular to the transverse line across the anterior superior iliac spine.

timeline was prepared by a research assistant who had no communication with the participants throughout the trial and was unaware of the study protocol. The randomization series (using block randomization) was created with SAS 9.4 statistical software with the participants equally likely to be assigned to each group (Figure 3). The generated code indicated group assignment and was sealed in an envelope until written informed consent was obtained.

Intervention procedures: There were two intervention groups in the study: one that received INIT with RRAF and another that received PRT. The groups received treatments by two independent physiotherapists (one assigned to each group) with expert knowledge of INIT and PRT. One of the physiotherapists (M.S.D.) had 6 years of clinical experience when the study took place and the other (M.A.F.) had 10 years of clinical experience at that time. To minimize investigator bias, the physiotherapists switched after 4 weeks of intervention. Participants attended two treatment sessions per week for 8 weeks. Additionally, each group also received stretching exercises (the same set of exercises given posttreatment as a home routine; Supplemental Material); these were performed immediately after the PRT or INIT treatment session (two exercise sessions per week for 8 weeks). 


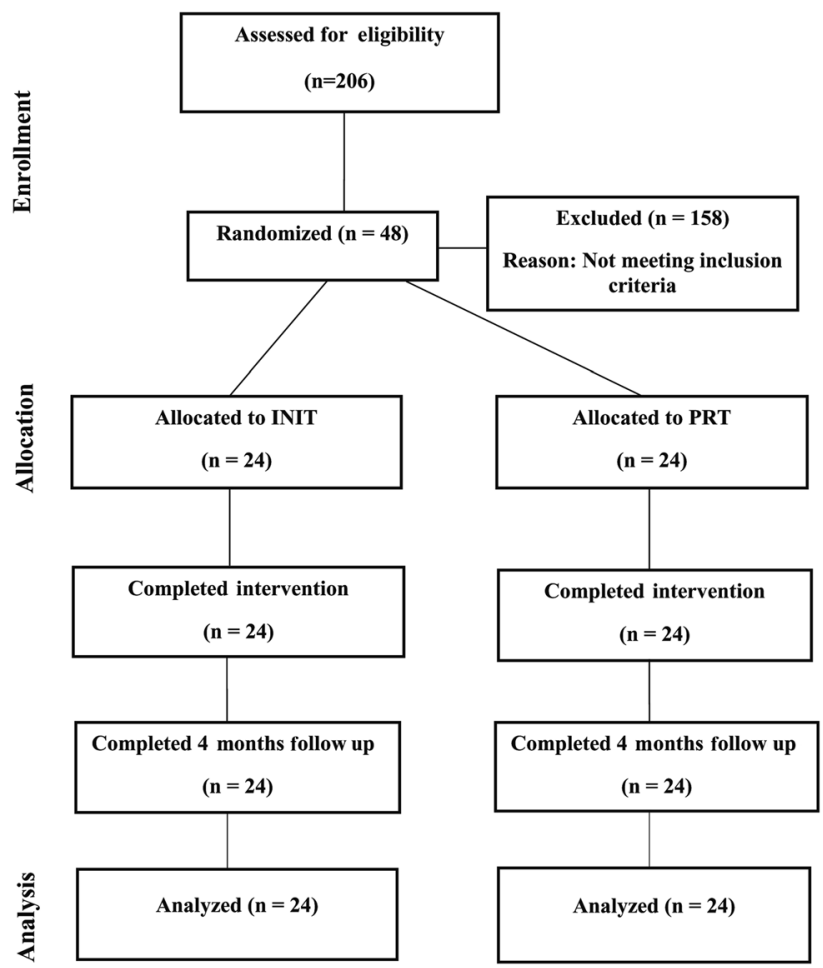

Figure 3: Flow chart of patients through the enrollment, allocation (randomization), and assessment processes in this study.

Integrated neuromuscular inhibition technique: INIT was applied using Chaitow's protocol [20,34]. A video demonstrating this technique is available in the Supplemental Material for this article. The participant was placed in a prone position with the hip flexed to approximately $60^{\circ}-90^{\circ}$ and abducted. The treated leg was suspended off the table with the bent knee resting on the therapist's thigh; the therapist was seated on the tender point side. The tender point or trigger point was palpated in the belly of the piriformis approximately halfway between the inferior lateral angle of the sacrum and the greater trochanter. Once the most dominant tender point or fasciculation (or both) was determined, the therapist then used one hand to apply an intermittent or sustained pressure with the pad(s) of the finger(s) at the location of the trigger point and maintained the pressure for 20-60 seconds (depending on participant's response to pain reduction). Fine tuning involving hip internal or external rotation by the therapist's other hand based on the location of the trigger point was applied to maintain the position of most ease (position of less sensitivity). When referred or local pain started to reduce in intensity, the compression treatment stopped and then the participant was instructed to use no more than $20 \%$ of available strength to attempt to take the limb away from the restriction barrier, while the therapist maintained his hands' positions and held the participant's limb firmly to create isometric contraction which was maintained for 7-10 seconds. Following the contraction, a reduction in tone was achieved in the piriformis and then the hypertonic or fibrotic fibers were further stretched and lengthened using Ruddy's reciprocal antagonist facilitation (RRAF) method. RRAF is a method of rapid pulsating contractions, termed rapid rhythmic resistive duction or pulsed MET, in which a patient introduces a series of miniature contractions or efforts (20 times in 10 seconds) against a therapist resistance [35]. Based on this premise, the participant was instructed to complete the INIT cycle by pushing his/her limb against the therapist resistance about 20 times in 10 seconds (two times per second), starting and stopping, so that no actual movement takes place, just a contraction and a relaxation, repetitively (no wobble and no bounce). The therapist instructed the participant to stop repetitive contractions after 10 seconds. Three repetitions of INIT were performed over 10 minutes at each clinical visit and participants were seen twice per week for 8 weeks, totaling 16 treatment sessions.

Positional release technique (PRT): PRT was applied using Speicher's protocol [18]. A video demonstrating this technique is available in the Supplemental Material for this article. The participant was placed in a prone position with the hip flexed to approximately $60^{\circ}-90^{\circ}$ and abducted. The treated leg was suspended off the table with the bent knee resting on the thigh of the therapist, who was seated on the tender point side. The location of the piriformis was examined by locating the coccyx with the fingers of one hand and the greater trochanter with the other hand. The tender point or trigger point was palpated in the belly of the piriformis approximately halfway between the inferior lateral angle of the sacrum and the greater trochanter. To properly identify the density of the piriformis, resistive hip external rotation was introduced. Once the most dominant tender point or fasciculation (or both) was determined, the therapist then used one hand to apply light pressure with the pad(s) of the finger(s) at the location of the trigger point and maintained the pressure for 2 minutes or until the pain subsided (determined by asking the participant to report a VAS score at 30 second intervals). Fine tuning involving hip internal or external rotation with the therapist's other hand based on the location of the trigger point was applied to maintain the position of most ease (position of less sensitivity). This procedure was repeated throughout the PRT treatment sessions until the participant's pain was reduced or nonexistent. Three repetitions of PRT were performed in 10 minutes at each clinical visit and participants were seen twice a week for 8 weeks, totaling 16 treatment sessions.

Stretching exercises: Participants in both groups were guided and instructed to perform stretching exercises (Supplemental Material); three repetitions of these exercises were performed over the course of 5 minutes at the end of each of the 16 clinical visits. These exercises were also prescribed to participants to perform at home twice per week over the course of the 4 months follow up period after the treatment period had ended.

Piriformis stretching: Participants were instructed to lie on their back with both knees bent and the foot of the unaffected leg flat on the table. The participant rested the ankle of the affected leg over the knee of the unaffected leg. The participant then grasped the thigh of the unaffected leg and pulled it toward the chest until a stretch was felt around the buttocks and along the outside of the hip on the affected leg. The stretch was held for 60 seconds, and then slowly returned to the starting position [11].

Hamstring/calf stretching: Participants were instructed to stand erect and lean forward with palms of both hands resting on the wall. The participant then stretched the hamstring and calf muscles of the affected lower limb by putting the foot of the affected limb behind and the foot of the unaffected limb front with both knees fully extended. When the heels of both lower limbs reached the floor, the knee of the front lower limb (the unaffected limb) was flexed forward toward the wall to increase the stretch on the soleus of the behind lower limb (the 
affected limb). The upper body was then swung left and right to ensure that the entire hamstring muscle group was activated and stretched [36].

\section{Adverse events}

Adverse (harmful or unpleasant) events reported by research participants were recorded in a standardized form by the investigators at all stages of data collection. This was done by asking the participants to report verbally any adverse events they had experienced after previous treatment contacts.

\section{Participant compliance}

Again, stretching exercises (Supplemental Material) were prescribed to the participants as a home regimen when the treatment period ended. These exercises were explained and taught in simple terms at the end of each treatment visit to ensure that participants received appropriate exercise instructions. Participants were also asked to suspend all pain medications to most accurately assess the efficacy of the manual techniques. Participants' compliance with this home regimen was assessed and reinforced every month via phone calls.

\section{Statistical analysis}

All data were analyzed using SPSS 23.0 (SPSS, Inc.). Descriptive statistics were used to summarize the demographics and clinical parameters of the participants. The Shapiro-Wilk test was used to assess the normality of the data, while the Levene test was used to assess the homogeneity of variances among groups. An independent t-test was used to compare measurable outcomes at baseline. The effect of INIT and PRT on pain, sciatica, functional mobility, and quality of life was examined using two way repeated measures analysis of variance (ANOVA) with the interventions (INIT and PRT) as the between-subject variable and time (baseline, immediately posttreatment, and 4 months posttreatment) as the within-subject variable. Differences between the means were considered at a $5 \%$ probability level $(\mathrm{p}<0.05)$ and the confidence interval (CI) value was set at $95 \%$.

\section{Results}

A total of 48 participants (age range, 25-47 years; mean age \pm standard deviation [SD], $32.81 \pm 3.27$ years) were enrolled in the study, with 24 participants each in the INIT and PRT treatment groups. Most patients (34; 70.8\%), were under the age of 35 years while seven (14.6\%) were 40 years or older. Thirty-nine $(81.2 \%)$ participants were male and nine (18.8\%) participants were female. All participants attended each treatment session and there were no dropouts. At the end of the 4 months follow up period, participants' adherence to the home routine was assessed via an in-person visits. Overall, 41 participants [85.4\%; 22 [91.7\%] in the PRT group and 19 [79.2\%] in the INIT group] were categorized as adherers to home regimen, while seven participants $(14.5 \%)$ were categorized as nonadherers. No significant between-group differences ( $p>0.05$ ) were observed in the baseline demographic and clinical variables of the participants (Table 1).

The Shapiro-Wilk test of normality (Table 2) showed that the distributions of all outcome variables were not significantly different from normal ( $p>0.05)$. Levene's test of equality of error variances indicated that there was homogeneity of variances of the three measurements (baseline, immediately posttreatment, and 4 months posttreatment) for all outcomes ( $>0.05)$ (Table 3). A repeated-measures ANOVA indicated that there was a significant time effect for all outcomes, with a significant interaction between time and intervention $(\mathrm{p}<0.001)$ (Table 4). The Bonferroni posthoc analyses of time and intervention effects indicated that the INIT group improved significantly better than the PRT group in all outcomes $(\mathrm{p}<0.05)$ immediately posttreatment (Table 5) and at 4 months posttreatment (Table 6).

Five participants $(10.4 \%$; four in the PRT group [16.7\%] and one in the INIT group [4.2\%]) reported a transient increase in pain within the first 24 hours of previous treatment sessions, which lasted for no more than 30 minutes. These adverse events were reported by the participants at the fourth (four patients) and fifth (one case) treatment sessions. No other adverse events were reported throughout the study.

Table 1: Demographic and clinical variables of the participants at baseline.

\begin{tabular}{lrrr}
\hline Variables & $\begin{array}{r}\text { INIT group } \\
\text { Mean (SD) } \\
(\mathbf{n = 2 4 )}\end{array}$ & $\begin{array}{r}\text { PRT group } \\
\text { Mean (SD) } \\
(\mathbf{n = 2 4 )}\end{array}$ & p-Value \\
\hline Sex & & & \\
$\quad$ Male, n (\%) & $21(87.5)$ & $18(75)$ & \\
Female, n (\%) & $3(12.5)$ & $6(25)$ & \\
Age, years & $33.27(3.29)$ & $32.98(3.76)$ & 0.765 \\
Height, m & $1.59(0.04)$ & $1.62(0.08)$ & 0.456 \\
Weight, kg & $57.23(12.08)$ & $58.01(12.03)$ & 0.189 \\
BMI, kg/m ${ }^{2}$ & $21.1(3.34)$ & $20.22(3.83)$ & 0.231 \\
VAS & $7.14(1.43)$ & $6.98(2.92)$ & 0.982 \\
SBI & $21.65(4.51)$ & $20.58(5.17)$ & 0.234 \\
TUG, s & $45.68(4.33)$ & $46.11(4.81)$ & 0.454 \\
SF-36 & $35.32(2.76)$ & $37.01(2.42)$ & 0.563 \\
Hip abduction, deg & $21.34^{\circ}(3.45)$ & $22.01^{\circ}(3.97)$ & 0.346 \\
Hip internal rotation, deg & $18.01^{\circ}(2.63)$ & $17.69^{\circ}(2.63)$ & 0.872 \\
\hline
\end{tabular}

BMI, body mass index; INIT, integrated neuromuscular inhibition technique; PRT, positional release therapy; SBI, sciatica bothersomeness index; SD, standard deviation; SF-36, short-form health survey-36; TUG, timed up and go test; VAS, visual analog scale. 
Table 2: Statistical test of normality for clinical variables (showing the test statistic and $p$ values at three timelines).

\begin{tabular}{|c|c|c|c|}
\hline Variables & Statistic value & Shapiro-Wilk test DF & p-Value \\
\hline \multicolumn{4}{|l|}{ VAS } \\
\hline Baseline & 0.761 & 48 & 0.938 \\
\hline Posttreatment & 0.725 & 48 & 0.069 \\
\hline 4 months follow up & 0.793 & 48 & 0.071 \\
\hline \multicolumn{4}{|l|}{ SBI } \\
\hline Baseline & 0.749 & 48 & 0.342 \\
\hline Posttreatment & 0.781 & 48 & 0.345 \\
\hline 4 months follow up & 0.733 & 48 & 1.245 \\
\hline \multicolumn{4}{|l|}{ TUG, seconds } \\
\hline Baseline & 0.723 & 48 & 0.098 \\
\hline Posttreatment & 0.743 & 48 & 0.087 \\
\hline 4 months follow up & 0.751 & 48 & 0.064 \\
\hline \multicolumn{4}{|l|}{ SF-36 } \\
\hline Baseline & 0.756 & 48 & 1.164 \\
\hline Posttreatment & 0.771 & 48 & 1.261 \\
\hline 4 months follow up & 0.796 & 48 & 0.889 \\
\hline \multicolumn{4}{|l|}{ Hip abduction $\left({ }^{0}\right)$} \\
\hline Baseline & 0.711 & 48 & 0.560 \\
\hline Posttreatment & 0.701 & 48 & 0.865 \\
\hline 4 months follow up & 0.726 & 48 & 0.583 \\
\hline \multicolumn{4}{|c|}{ Hip internal rotation $\left({ }^{0}\right)$} \\
\hline Baseline & 0.760 & 48 & 1.162 \\
\hline Posttreatment & 0.761 & 48 & 0.666 \\
\hline 4 months follow up & 0.716 & 48 & 0.977 \\
\hline
\end{tabular}

DF, degree of freedom; INIT, integrated neuromuscular inhibition technique; PRT, positional release therapy; SBI, sciatica bothersomeness index; SF-36, short form health survey-36; TUG, timed up and go test; VAS, visual analog scale.

\section{Discussion}

This study examined the effect of INIT compared with PRT in the management of PS. The findings of this study indicated that there was a significant time effect for all outcomes (pain, hip range of motion, sciatica, functional mobility, and quality of life), with a significant interaction between time and intervention. These findings indicated that all outcomes significantly improved over time (from baseline to 4 months posttreatment) and the effect of time on each outcome depended on the intervention received. The findings of this study are similar to those of Mujawar et al. [23], who reported that the INIT was effective in the management of PS. Additionally, a study by Senthilkumar et al. [9] also reported that PRT was effective in the management of PS.

The findings of this study also indicated that INIT was more effective than PRT in the management of PS in all outcomes and at all recorded timelines (immediately
Table 3: Levene's test of equality of error variances.

\begin{tabular}{|c|c|c|c|c|}
\hline Variables & $\boldsymbol{F}$ & DF1 & DF2 & p-Value \\
\hline \multicolumn{5}{|l|}{ VAS } \\
\hline Baseline & 0.522 & 1 & 46 & 0.392 \\
\hline Posttreatment & 0.073 & 1 & 46 & 0.853 \\
\hline 4 months follow up & 4.63 & 1 & 46 & 0.912 \\
\hline \multicolumn{5}{|l|}{ SBI } \\
\hline Baseline & 0.134 & 1 & 46 & 0.765 \\
\hline Posttreatment & 3.345 & 1 & 46 & 0.123 \\
\hline 4 months follow up & 2.389 & 1 & 46 & 0.122 \\
\hline \multicolumn{5}{|l|}{ TUG, seconds } \\
\hline Baseline & 3.345 & 1 & 46 & 0.564 \\
\hline Posttreatment & 5.361 & 1 & 46 & 0.634 \\
\hline 4 months follow up & 0.589 & 1 & 46 & 0.444 \\
\hline \multicolumn{5}{|l|}{ SF-36 } \\
\hline Baseline & 5.674 & 1 & 46 & 0.437 \\
\hline Posttreatment & 3.398 & 1 & 46 & 0.743 \\
\hline 4 months follow up & 0.262 & 1 & 46 & 0.111 \\
\hline \multicolumn{5}{|l|}{ Hip abduction $\left({ }^{0}\right)$} \\
\hline Baseline & 4.012 & 1 & 46 & 0.557 \\
\hline Posttreatment & 6.567 & 1 & 46 & 0.678 \\
\hline 4 months follow up & 4.933 & 1 & 46 & 0.345 \\
\hline \multicolumn{5}{|c|}{ Hip internal rotation $\left({ }^{0}\right)$} \\
\hline Baseline & 2.309 & 1 & 46 & 0.611 \\
\hline Posttreatment & 7.577 & 1 & 46 & 0.222 \\
\hline 4 months follow up & 5.899 & 1 & 46 & 0.788 \\
\hline
\end{tabular}

DF, degree of freedom; F, analysis of variance; INIT, integrated neuromuscular inhibition technique; PRT, positional release therapy; SBI, sciatica bothersomeness index; SF-36, short form health survey 36 ; TUG, timed up and go test; VAS, visual analog scale.

posttreatment and 4 months posttreatment). While similar findings were reported in previous studies [9, 23], those studies reported shorter term follow up periods - immediately after a single treatment session [23] and after seven treatment sessions across 2 weeks [9]. Additionally, those studies [9, 23] did not use valid diagnostic criteria for PS [5] nor did they report intermediate or long term follow up data, which provides further contrast with our current study. Although some studies [10, 11, 21, 22] have reported the effectiveness of MET in the management of PS, these studies did not use the MET in form of INIT. These findings indicate that our current study is distinctive and supports the effectiveness of INIT in the management of patients with PS.

The significant improvement achieved in the INIT group in our study may be related to the dual benefit of both PRT and MET administered simultaneously in the form of INIT. PRT has been proposed to relieve pain and muscle spasms by producing reactive hyperemia in the treated tissues, or from the spinal reflex mechanism [19]. MET has been proposed to produce muscle relaxation and 
Table 4: Two way repeated measures analysis of variance for between group and time interactions (showing the ANOVA, degree of freedom and $p$-values of between group and time interactions).

\begin{tabular}{|c|c|c|}
\hline Variable & $F$, DF & p-Value \\
\hline \multicolumn{3}{|l|}{ VAS } \\
\hline Group & $25.374(1.46)$ & $<0.001$ \\
\hline Time & $67.333(1.92)$ & $<0.001$ \\
\hline Group*Time & $221.785(2.92)$ & $<0.001$ \\
\hline \multicolumn{3}{|l|}{ SBI } \\
\hline Group & $114.223(1.46)$ & $<0.001$ \\
\hline Time & $29.478(1.92)$ & $<0.001$ \\
\hline Group^Time & $61.372(2.92)$ & $<0.001$ \\
\hline \multicolumn{3}{|l|}{ TUG, seconds } \\
\hline Group & $98.978(1.46)$ & $<0.001$ \\
\hline Time & $156.635(1.92)$ & $<0.001$ \\
\hline Group^Time & $27.559(2.92)$ & $<0.001$ \\
\hline \multicolumn{3}{|l|}{ SF-36 } \\
\hline Group & $46.667(1.46)$ & $<0.001$ \\
\hline Time & $223.468(1.92)$ & $<0.001$ \\
\hline Group*Time & $123.681(2.92)$ & $<0.001$ \\
\hline \multicolumn{3}{|c|}{ Hip abduction, $\operatorname{deg}\left({ }^{0}\right)$} \\
\hline Group & $227.012(1.46)$ & $<0.001$ \\
\hline Time & $69.455(1.92)$ & $<0.001$ \\
\hline Group^Time & $119.908(2.92)$ & $<0.001$ \\
\hline \multicolumn{3}{|c|}{ Hip internal rotation, $\operatorname{deg}\left({ }^{0}\right)$} \\
\hline Group & $43.633(1.46)$ & $<0.001$ \\
\hline Time & $181.674(1.92)$ & $<0.001$ \\
\hline Group*Time & $209.197(2.92)$ & $<0.001$ \\
\hline
\end{tabular}

DF, degree of freedom; F, analysis of variance; INIT, integrated neuromuscular inhibition technique; PRT, positional release therapy; SBI, sciatica bothersomeness index; SF-36, short form health survey 36; TUG, timed up and go test; VAS, visual analog scale.

lengthening by decreasing muscle tone that happens due to the effects of stretch receptors (Golgi tendon organs), which react to excessive stretching of the muscles by counteracting longer muscle contractions; this has a lengthening effect due to the immediate relaxation of the whole muscle under tension [20]. It has been thought that these effects of INIT are due to the integration of both PRT and MET simultaneously and have significantly assisted in the restoration of pathological changes of the piriformis while also lessening compressive forces on the sciatic nerve [20].

Another possible reason for the greater improvements recorded by the INIT group could have been the effect of the INIT protocol on the hip musculature. The integration of the RRAF method into the INIT protocol activated hip musculature (by stretching and strengthening) while producing an automatic reciprocal inhibition of the piriformis [34]. Studies [11, 31, 37, 38] have indicated that therapies targeting hip musculature may have the ability to relieve piriformis pain. Given that the piriformis attaches to the greater trochanter, excessive hip motion may put a strain on this muscle resulting in compression of the sciatic nerve [31]. We hypothesized that if the hip musculature could be stretched and strengthened by introducing the RRAF method into the INIT protocol, the strain on the piriformis could be minimized through antagonist activity (prevention of undue hip motion), resulting in less compression on the sciatic nerve. While a previous study [23] did investigate the effect of INIT in the management of individuals with PS, it did not integrate the RRAF method into the INIT protocol as was done in the current study.

The significant improvement achieved in both groups may also be related to the effect of stretching exercises that were used as adjunct therapies. Piriformis stretching has been indicated to improve muscle spasm, length, and flexibility, which may ultimately relieve pain and pressure on the sciatic nerve $[11,39]$. Previous studies [12, 4042] have shown the effectiveness of stretching exercises in reducing pain and sciatica among individuals with PS, but these exercises should not replace INIT or PRT because they operate via different mechanisms $[11,19,20,34,39,43]$. However, the greater improvement achieved by the INIT group in this current study could indicate that the stretching exercises (which were performed by all participants) did not have a meaningful effect on the results.

It is worthy of note that a few participants experienced a transient increase in pain following the INIT and PRT treatment sessions at some point in time; however, this pain subsided within 30 minutes of onset. Transient increases in pain following neuromuscular and trigger point release techniques are very common and were reported in a previous study of lumbar disc herniation patients who experienced transient pains following application of the progressive inhibition of neuromuscular structures technique [36]. Similar occurrences were also reported by Chaitow [34, 35] and Dowling [44] as a normal tissue physiologic response to treatment.

\section{Limitations}

This study did not use a control group to test the efficacy of INIT or PRT in the management of PS. This study also did not assess any psychosocial barriers to recovery that may be associated with chronic pain. Additionally, the physical function subscale of the SF-36 was not completed to 
Table 5: Mean baseline to immediately posttreatment change scores for clinical outcomes.

\begin{tabular}{|c|c|c|c|c|c|}
\hline Variable & Baseline (SD) & $\begin{array}{r}\text { Immediately } \\
\text { posttreatment (SD) }\end{array}$ & $\begin{array}{r}\text { Within-group } \\
\text { change score (SD) }\end{array}$ & $\begin{array}{r}\text { Between-group } \\
\text { change score }(95 \% \mathrm{Cl})\end{array}$ & p-Value \\
\hline VAS & & & & $4.27(3.33-4.78)$ & 0.001 \\
\hline INIT group & $7.14(1.43)$ & $2.11(0.11)$ & $5.03(1.22)$ & & \\
\hline PRT group & $6.98(2.92)$ & $3.41(0.32)$ & $3.56(1.68)$ & & \\
\hline SBI & & & & $5.74(5.21-7.99)$ & 0.011 \\
\hline INIT group & $21.65(4.51)$ & $9.33(2.03)$ & $11.99(0.55)$ & & \\
\hline PRT group & $20.58(5.17)$ & $13.72(2.22)$ & $6.88(1.02)$ & & \\
\hline TUG, seconds & & & & $6.43(4.66-10.11)$ & 0.013 \\
\hline INIT group & $45.68(4.33)$ & $17.61(2.45)$ & $28.17(3.20)$ & & \\
\hline PRT group & $46.11(4.81)$ & $23.02(2.64)$ & $23.09(4.50)$ & & \\
\hline SF-36 & & & & $8.69(5.73-9.05)$ & 0.001 \\
\hline INIT group & $35.32(2.76)$ & $77.98(3.51)$ & $42.66(5.37)$ & & \\
\hline PRT group & $37.01(2.42)$ & $63.41(3.69)$ & $26.47(6.61)$ & & \\
\hline Hip abduction $\left({ }^{0}\right)$ & & & & $7.03(5.00-8.89)$ & 0.001 \\
\hline INIT group & $21.34(3.45)$ & $31.67(4.51)$ & $10.33(2.81)$ & & \\
\hline PRT group & $22.01(3.97)$ & $28.45(4.79)$ & $6.44(2.43)$ & & \\
\hline Hip internal rotation $\left(^{0}\right)$ & & & & $8.11(7.71-8.99)$ & 0.001 \\
\hline INIT group & $18.01(2.63)$ & $29.02(3.81)$ & $11.01(3.33)$ & & \\
\hline PRT group & $17.69(2.63)$ & $26.43(4.35)$ & $8.74(4.01)$ & & \\
\hline
\end{tabular}

$\mathrm{Cl}$, confidence interval; INIT, integrated neuromuscular inhibition technique; PRT, positional release therapy; SBI, sciatica bothersomenesS index; SF-36, short form health survey-36; SD, standard deviation; TUG, timed up and go test; VAS, visual analog scale.

Table 6: Mean change scores in clinical outcomes from immediately posttreatment to 4 months follow up.

\begin{tabular}{|c|c|c|c|c|c|}
\hline Variable & $\begin{array}{r}\text { Immediately } \\
\text { posttreatment (SD) }\end{array}$ & $\begin{array}{r}4 \text { months } \\
\text { follow up (SD) }\end{array}$ & Within-group change score (SD) & $\begin{array}{r}\text { Between-group } \\
\text { change score }(95 \% \mathrm{Cl})\end{array}$ & p-Value \\
\hline VAS & & & & $2.34(1.77-.13)$ & 0.014 \\
\hline INIT group & $2.11(0.11)$ & $0.34(0.02)$ & $1.77(0.23)$ & & \\
\hline PRT group & $3.41(0.32)$ & $1.56(0.11)$ & $1.86(0.77)$ & & \\
\hline SBI & & & & $4.19(3.01-6.21)$ & 0.031 \\
\hline INIT group & $9.33(2.03)$ & $2.11(1.02)$ & $7.22(2.03)$ & & \\
\hline PRT group & $13.72(2.22)$ & $5.98(1.77)$ & $7.74(2.58)$ & & \\
\hline TUG, seconds & & & & $8.72(5.98-11.91)$ & 0.001 \\
\hline INIT group & $17.61(2.45)$ & $6.54(1.79)$ & $11.07(1.96)$ & & \\
\hline PRT group & $23.02(2.64)$ & $11.33(2.06)$ & $11.68(2.13)$ & & \\
\hline SF-36 & & & & $12.34(8.37-14.48)$ & 0.001 \\
\hline INIT group & $77.98(3.51)$ & $91.04(4.33)$ & $13.06(4.18)$ & & \\
\hline PRT group & $63.41(3.69)$ & $79.61(4.67)$ & $16.21(2.69)$ & & \\
\hline Hip abduction ( $\left.{ }^{0}\right)$ & & & & $10.33(7.09-11.33)$ & 0.001 \\
\hline INIT group & $31.67(4.51)$ & $38.97(2.24)$ & $7.51(2.07)$ & & \\
\hline PRT group & $28.45(4.79)$ & $33.39(2.51)$ & $4.95(3.00)$ & & \\
\hline Hip internal rotation $\left({ }^{0}\right)$ & & & & $8.88(5.00-9.66)$ & 0.001 \\
\hline INIT group & $29.02(3.81)$ & $37.03(3.99)$ & $8.02(2.11)$ & & \\
\hline PRT group & $26.43(4.35)$ & 31.09 (3.77) & $4.66(2.03)$ & & \\
\hline
\end{tabular}

$\mathrm{Cl}$, confidence interval; INIT, integrated neuromuscular inhibition technique; PRT, positional release therapy; SBI, sciatica bothersomenesS index; SF-36, short form health survey-36; SD, standard deviation; TUG, timed up and go test; VAS, visual analog scale.

determine whether there were any differences in the quality of life findings. Further, the stretching exercises used immediately posttreatment at each session and over the 4 months of posttreatment follow up were a potential confounding variable in this study. Future studies may be conducted to address these concerns. 


\section{Clinical relevance to osteopathic medicine}

OMT is a set of hands on techniques used by osteopathic physicians (DOs) to diagnose, treat, and prevent illness or injury [45]. While OMT is typically performed by a DO, manipulative therapy in this study was not performed by a DO; however, similarities may be appreciated. The current study demonstrated the therapeutic efficacy of INIT and PRT as an indirect OMT approach in the management of PS. The application of both INIT and PRT involved the palpation of trigger points along a muscular structure and then applying ischemic compression to deactivate those trigger points. Both INIT and PRT are very similar to other indirect OMT techniques such as progressive inhibition of neuromuscular structures (PINS) [36, 44], except that PRT and MET are simultaneously integrated into the INIT protocol while trigger points are progressively or sequentially deactivated along a neuromuscular continuum in the PINS protocol.

\section{Conclusions}

INIT was more effective than PRT in the management of individuals with PS. It should be noted the significant improvement achieved in both the groups may have also been contributed to by the effect of stretching exercises, which were used as adjunct therapies by both groups.

Research funding: None reported.

Author contributions: All authors provided substantial contributions to conception and design, acquisition of data, or analysis and interpretation of data; Mr Danazumi drafted the article and revised it critically for important intellectual content; all authors gave final approval of the version of the article to be published; and all authors agree to be accountable for all aspects of the work in ensuring that questions related to the accuracy or integrity of any part of the work are appropriately investigated and resolved.

Competing interests: None reported.

Informed consent: Written informed consent was obtained from the participants and they were assured of their rights to withdraw from the study at any point in time and their full anonymity was maintained.

Ethical approval: Ethical approval to conduct this study was obtained from the Health Research Ethics Committee of Federal Medical Centre (FMC), Nguru, Yobe State, Nigeria (Registration number: FMC/N/CL.SERV/355/VOL IV/146). The trial was registered with the Pan African Clinical Trial Registry (PACTR201911583499287).

\section{References}

1. Boyajian-O'Neill LA, McClain RL, Coleman MK, Thomas PP. Diagnosis and management of piriformis syndrome: an osteopathic approach. J Am Osteopath Assoc 2008;108:657-64.

2. Fishman LM, Dombi GW, Michaelsen C, Ringel S, Rozbruch J, Rosner B, et al. Piriformis syndrome: diagnosis, treatment, and outcome-a 10-year study [review]. Arch Phys Med Rehabil 2002; 83:295-301.

3. Singh US, Meena RK, Singh CK, Singh AJ, Singh AM, Langshong R. Prevalence of piriformis syndrome among the cases of low back/ buttock pain with sciatica: a prospective study. J Med Soc 2013; 27:94-9.

4. Miller T, White K, Ross D. The diagnosis and management of piriformis syndrome: myths and facts. Can J Neurol Sci 2012;39: 577-83.

5. Hopayian K, Danielyan A. Four symptoms define the piriformis syndrome: an updated systematic review of its clinical features. Eur J Orthop Surg Traumatol 2018;28:155-64.

6. Khuman PR, Surbala L, Dusad G, Jadeja S, Chandrabharu V. Effect of reciprocal inhibition muscle energy technique in acute piriformis syndrome: a single case study. Int J Recent Sci Res 2014;5:1794-8.

7. Misirlioglu TO, Akgun K, Palamar D, Erden MG, Erbilir T. Piriformis syndrome: comparison of the effectiveness of local anesthetic and corticosteroid injections: a double-blinded, randomized controlled study. Pain Physician 2015;18:163-71.

8. Alsaeid Ahmed MA. Dexamethasone vs. magnesium sulfate as an adjuvant to local anesthetics in the ultra-sound guided injection of piriformis muscle for the treatment of piriformis syndrome. Open Anesthesiol J 2020;14:35-41.

9. Senthilkumar S, Fathima A, Keerthana S. A study to evaluate the effectiveness of positional release therapy in piriformis syndrome. Int J Recent Sci Res 2019;10:35163-7.

10. Nambi G. Effect of reciprocal inhibition and post isometric relaxation; types of muscle energy technique in piriformis syndrome - a comparative study. Phys Med Rehabil Res 2018;3: 1-5.

11. Park JC, Shim JH, Chung SH. The effects of three types of piriform muscle stretching on muscle thickness and the medial rotation angle of the coxal articulation. J Phys Ther Sci 2017;29:1811-4.

12. Ijaz Gondal MJ, Iqbal MA, Nasir RH, Tabassum R, Rasul A. Study of treatment outcome of piriformis syndrome with and without physiotherapy treatment. Ann King Edward Med Univ 2015;21: 78-88.

13. Aquino-Jose VM, Blinder V, Johnson J, Havryliuk T. Ultrasoundguided trigger point injection for piriformis syndrome in the emergency department. J Am Coll Emerg Phys Open 2020;1: 876-9.

14. Fishman LM, Wilkins AN, Rosner B. Electrophysiologically identified piriformis syndrome is successfully treated with incobotulinum toxin and physical therapy. Muscle Nerve 2017;56: 258-63.

15. Han SK, Kim YS, Kim TH, Kang SH. Surgical treatment of piriformis syndrome. Clin Orthop Surg 2017;9:136-44.

16. Kale A, Basol G, Kuru B, Gundogdu E, Mat E, Yildiz G, et al. Laparoscopic surgical approach for the treatment of pelvic piriformis syndrome. Authorea 2020. https://doi.org/10.22541/ au.160372848.88093896/v1. 
17. Hogan E, Vora D, Sherman JH. A minimally invasive surgical approach for the treatment of piriformis syndrome: a case series. Chin Neurosurg J 2020;6:8.

18. Speicher T. Clinical guide to positional release therapy. Med. Sci. Sport. Exerc; 2017. Available from: https://www.human-kinetics. co.uk/9781450496247/clinical-guide-to-positional-releasetherapy/ [Accessed 10 Jan 2021].

19. Hou CR, Tsai LC, Cheng KF, Chung KC, Hong CZ. Immediate effects of various physical therapeutic modalities on cervical myofascial pain and trigger-point sensitivity. Arch Phys Med Rehabil 2002; 83:1406-14.

20. Chaitow L, Franke H, Chaitow L. Muscle energy techniques, 4th ed. United Kingdom: Churchill Livingstone/Elsevier; 2013.

21. Jain E, Moharkar A, Golhar S. Effectiveness of muscle energy technique \& neural tissue mobilization on buttock pain, hip internal rotation \& functional disability in female patients with chronic non-specific low back pain having piriformis syndrome at the end of 10 sessions: a comparative study. Int J Appl Res 2020; 6:433-42.

22. Kutty NN, Siddeeque S, Azharuddin TS, Othayoth N, Bineesh CP. Effect of muscle energy technique with deep friction massage on pain, disability and internal rotation range of motion of hip joint in individuals with piriformis syndrome. Indian J Physiother Occup Ther 2020;14:148-53.

23. Mujawar JC, Chotai K, Kanase S, Jadhav A. Effectiveness of neuromuscular therapy and active release technique in young adults with piriformis tightness. Indian J Physiother Occup Ther 2019;13:212-7.

24. Suresh K, Chandrashekara S. Sample size estimation and power analysis for clinical research studies. J Hum Reprod Sci 2015;8: 186.

25. Hughes S, Goldstein M, Hicks D, Pelligrini V. Extrapelvic compression of the sciatic nerve. J Bone Joint Surg 1992;74-A: 1553-9.

26. Kay J, de Sa D, Morrison L, Fejtek E, Simunovic N, Martin HD, et al. Surgical management of deep gluteal syndrome causing sciatic nerve entrapment: a systematic review. Arthroscopy 2017;33: 2263-78.e1.

27. Kizaki K, Uchida S, Shanmugaraj A, Aquino CC, Duong A, Simunovic N, et al. Deep gluteal syndrome is defined as a nondiscogenic sciatic nerve disorder with entrapment in the deep gluteal space: a systematic review. Knee Surg Sports Traumatol Arthrosc 2020;28:3354-64.

28. Boonstra AM, Schiphorst Preuper HR, Reneman MF, Posthumus JB, Stewart RE. Reliability and validity of the visual analog scale for disability in patients with chronic musculoskeletal pain. Int J Rehabil Res 2008;31:165-9.

29. Gautschi OP, Smoll NR, Corniola MV, Joswig H, Chau I, Hildebrandt $\mathrm{G}$, et al. Validity and reliability of a measurement of objective functional impairment in lumbar degenerative disc disease: the timed up and go (TUG) test. Neurosurgery 2016;79: 270-8.

30. Haugen AJ, Grøvle L, Brox JI, Natwig B, Keller A, Soldal D, et al. Estimates of success in patients with sciatica due to lumbar disc herniation depend upon outcome measure. Eur Spine J 2011;20: 1669-75.

31. Tonley JC, Yun SM, Kochevar RJ, Dye JA, Farrokhi S, Powers $\mathrm{CM}$. Treatment of an individual with piriformis syndrome focusing on hip muscle strengthening and movement reeducation: a case report. J Orthop Sports Phys Ther 2010; 40:103-11.

32. Hoffman DL, Dukes EM. The health status burden of people with fibromyalgia: a review of studies that assessed health status with the SF-36 or the SF-12. Int J Clin Pract 2008;62: 115-26.

33. Nussbaumer S, Leunig M, Glatthorn JF, Stauffacher S, Gerber H, Maffiuletti NA. Validity and test-retest reliability of manual goniometers for measuring passive hip range of motion in femoroacetabular impingement patients. BMC Muscoskel Disord 2010;11:194.

34. Chaitow L, DeLany J. Clinical application of neuromuscular techniques. The lower body, 2nd ed. United Kingdom: Churchill Livingstone; 2011, 2.

35. Chaitow L, DeLany J. Clinical application of neuromuscular techniques. The upper body, 2nd ed. United Kingdom: Churchill Livingstone; 2008, vol 1, chapter 10:212 p.

36. Bello B, Danazumi MS, Kaka B. Comparative effectiveness of 2 manual therapy techniques in the management of lumbar radiculopathy: a randomized clinical trial. J Chiropr Med 2019;18: 253-60.

37. Laha K, Sarkar B, Kumar P, Patel L, Sarkar N. Efficacy of hip abductor and extensor strengthening on pain, strength and lower extremity function in piriformis syndrome: a randomized clinical trial. Int J Health Sci Res 2018;8:80-8.

38. Lim J, Lee I, Kim D. A movement-system-impairment approach to the evaluation and treatment of a patient with piriformis syndrome: a case report. J Musculoskelet Sci Technol 2018;2: 43-9.

39. Gulledge BM, Marcellin-Little DJ, Levine D, Tillman L, Harrysson OLA, Osbprne JA, et al. Comparison of two stretching methods and optimization of stretching protocol for the piriformis muscle. Med Eng Phys 2014;36:212-8.

40. Kukadia HA, Malshikare A, Palekar JT. Effect of passive stretching $\mathrm{v} / \mathrm{s}$ myofascial release in improving piriformis flexibility in females - a comparative study. Indian J Physiother Occup Ther 2019;13:57-61.

41. Sarmitha R, Subramaniam SS. The effectiveness of myofascial release over stretching on pain and range of motion among female college students with piriformis syndrome. Malays J Mov Health Exerc 2020;9:45-56.

42. Saeed Q, Malik AN, Ghulam S. Outcome of specific piriformis stretching technique in females with piriformis syndrome. J Pioneer Med Sci 2017;7:55-8.

43. Danazumi MS, Yakasai AM, Ibrahim SU. Effect of integrated neuromuscular inhibition technique in the management of piriformis syndrome: a case report. Middle East J Rehabil Health Stud 2020;7:e101764.

44. Dowling DJ. Progressive inhibition of neuromuscular structures (PINS) technique. J Am Osteopath Assoc 2000;100:285-6, 289-98.

45. American Osteopathic Association. What is osteopathic medicine? 2021. Available from: https://osteopathic.org/whatis-osteopathic-medicine/osteopathic-manipulative-treatment/ [Accessed 22 Apr 2021].

Supplemental Material: The online version of this article offers supplementary material (https://doi.org/10.1515/jom-2020-0327). 